\title{
ADAPTACIÓN Y VALIDACIÓN DEL CUESTIONARIO DE SUPERVISIÓN ABUSIVA DE TEPPER (SAT) EN EMPLEADOS DE LA REGIÓN DE ARICA Y PARINACOTA
}

\author{
ADAPTATION AND VALIDATION OF THE TEPPER ABUSE SUPERVISION \\ QUESTIONNAIRE (SAT) IN EMPLOYEES OF THE ARICA \\ AND PARINACOTA REGION
}

\author{
Patricio Mena-Chamorro* \\ Universidad de Tarapacá \\ Arica-Chile \\ Rodrigo Ferrer-Urbina \\ Universidad de Tarapacá \\ Arica-Chile \\ Francisca Parra \\ Universidad de Tarapacá \\ Arica-Chile \\ María José Ron \\ Universidad de Tarapacá \\ Arica-Chile \\ Javiera Vásquez \\ Universidad de Tarapacá \\ Arica-Chile \\ Recibido junio de 2020/Received June, 2020 \\ Aceptado mayo de 2021/Accepted May, 2021
}

\begin{abstract}
RESUMEN
El propósito de este estudio fue adaptar y obtener evidencia de validez del cuestionario de Supervisión Abusiva de Tepper (SAT) para su uso en adultos trabajadores de Arica, Chile. Se utilizó un diseño transversal de tipo instrumental, con una muestra conformada por 287 participantes provenientes de empresas privadas de la ciudad. Se realizaron estimaciones de fiabilidad mediante coeficiente omega jerárquico de McDonald's y alfa de Cronbach y se obtuvo evidencias de validez basada en la estructura interna del test, mediante análisis factoriales confirmatorios. Los resultados mostraron que la versión dicotomiza depurada proporciona adecuados indicadores de ajuste comparativos y absolutos (RMSEA = .04; CFI = .99; TLI = .98), con adecuados niveles de fiabilidad $(\alpha=.87 ; \omega=.88)$. Se concluye que la escala de Supervisión Abusiva de Tepper (SAT) presenta propiedades psicométricas adecuadas para la medición de supervisión abusiva en muestras equivalentes de adultos trabajadores.
\end{abstract}

Palabras Clave: Supervisión abusiva, Evidencia de validez, Fiabilidad, Adaptación de escalas psicométricas. 


\section{ABSTRACT}

The purpose of this study was to adapt and obtain evidence of validity of the Tepper Abusive Supervision questionnaire (TAS) for use in adult workers in Arica, Chile. An instrumental cross-sectional design was used, with a sample of 287 participants from private companies in the city. Reliability estimates were performed by means of McDonald's omega hierarchical coefficient and Cronbach's alpha and validity evidence was obtained based on the internal structure of the test, by means of confirmatory factor analysis. The results showed that the debugged dichotomous version provides adequate comparative and absolute fit indicators $(R M S E A=.04 ; C F I=.99 ; T L I=.98)$, with adequate levels of reliability $(\alpha=.87 ; \omega=.88)$. It is concluded that the Tepper Abusive Supervision questionnaire (TAS) shows adequate psychometric properties for the measurement of abusive supervision in equivalent samples of working adults.

Key Words: Abusive supervision, Evidence of validity, Reliability, Adaptation of psychometric scales.

\section{INTRODUCCIÓN}

La relación supervisor-colaborador implica una relación mutua, en la que se espera que los supervisores tengan conductas como designar tareas, clarificar responsabilidades, resolver problemas y motivar a los trabajadores para cumplir con lo planificado (Ahmad and Ibrahim, 2015; De Jong and Den Hartog, 2007). No es infrecuente que algunos supervisores manifiesten conductas indeseadas que impactan negativamente en los resultados organizacionales, así como los arrebatos incontrolados, falta de reconocimiento a los colaboradores, incumplimiento de promesas, criticar en público, mentir, resaltar los errores de los subordinados, entre otros (Martinko, Harvey, Sikora and Douglas, 2011; Contreras, Barbosa, Juárez, Uribe y Mejía, 2009; Tepper, 2000; 2007). Para referirse a estas conductas hostiles, en la literatura se han ocupado diversos conceptos, como líderes tóxicos (Burke, 2017), destructivos (Burns, 2017), narcisistas (Krasikova, et al., 2013), pequeña tiranía (Boddy, 2011) o psicópatas corporativos (Rosenthal and Pittinsky, 2006; Ashforth, 1994), sin embargo en los últimos años la mayoría de los estudios en el área organizacional han optado por utilizar el constructo de supervisión abusiva, introducido por Tepper (2000), y que refiere a una percepción subjetiva, por parte de los subordinados, respecto del grado en que su supervisor efectúa o manifiesta conductas hostiles que no impliquen contacto físico. La supervisión abusiva se caracteriza específicamente por dos condiciones: (1) poseer una relación de asimetría de poder; y (2) el uso de esa posición en menoscabo de algún subordinado.

En la actualidad, Chile presenta una tasa de ocupación nacional del $57,5 \%$, que ha ido decreciendo durante los últimos doce meses, repercutiendo directamente en el aumento de la tasa de desocupación $(10,3 \%)$ y ocupación informal (27\%) (Instituto Nacional de Estadística [INE], 2021). Si bien los valores laborales en Chile están asociados con aspectos históricos y culturales del país, como el compadrazgo, legalismo y la relación patrón-inquilino en la hacienda, según los estudios realizados con el modelo Hosftede para la evaluación de culturas nacionales (para una revisión, ver Hofstede and Minkov, 2010), han caracterizado a Chile por manifestar una cultura organizacional en la que predomina un fuerte sentido de jerarquía, individualismo y evitación de la incertidumbre (Gómez y Rodríguez, 2006). En este sentido, los subordinados asumen acríticamente las opiniones e instrucciones de sus jefes directos y aceptan ser supervisados. Además, el estilo de administración chileno al favorecer las conductas individualistas con incentivos económicos, reconocimiento y participación, promueve relaciones entre pares competitivas y conflictivas (Didier y Luna, 2017).

En los últimos años, el interés por investigar la supervisión abusiva ha aumentado sistemáticamente (Mackey, Frieder, Bree and Martinko, 2017; Zhang and Bednall, 2015), aspecto que se ve reflejado en la gran cantidad de estudios que proponen a las conductas hostiles como una variable importante que influye en el rendimiento, abandono laboral, salud mental y bienestar de los subordinados (Tepper, Simon and Park, 2017; Zhang and Liao, 2015; Rodwell, Brunetto, Demir, Shacklock and FarrWharton, 2014). La supervisión abusiva presenta múltiples perjuicios en el desempeño global de las personas y las organizaciones, propiciando mayores niveles de estrés, sintomatología depresiva, ansiedad y un menor rendimiento laboral en los trabajadores, así como un mayor ausentismo y rotación de personal (Palomino, Espinoza y Arellano, 2013; Farh and Chen, 2014; Khan, Qureshi and Ahmad 2010). Un estudio en empleados chinos mostró una asociación negativa entre la supervisión abusiva y el desempeño laboral de los empleados, que además estaba mediada por la justicia del liderazgo, la confianza del supervisor y la autoeficacia (Chen and Wang, 
2017). Otro estudio en empleados canadienses mostró que la supervisión abusiva estaba asociada con niveles más altos de angustia y problemas en la relación trabajo-familia (Zhou, Marchand and Guay, 2018). Un estudio transcultural evidenció que la cultura posee un efecto moderador en la relación de supervisión abusiva y justicia interpersonal, además de mostrar que los subordinados de la cultura anglosajona (frecuentemente caracterizada por ser individualista) perciben de forma más intensa los efectos negativos de estas conductas que los de la cultura asiática (frecuentemente caracterizada por ser colectivista) (Vogel et al., 2015).

Si bien la importancia y repercusión de las conductas hostiles en las organizaciones está fundamentada en la evidencia científica disponible, la forma en que se miden estas conductas ha sido controversial, ya que se han utilizado diversas tareas experimentales (e.g. Brees, Martinko and Harvey, 2016; Liang et al., 2016) o escalas de autorreporte (e.g. escala de Liderazgo Tóxico, Schmidt, 2008; Inventario de la personalidad narcisista, Raskin and Hall, 1979; escala de Pequeña Tiranía, Ashforth, 1987), lo que en muchas ocasiones dificulta el contraste y replicabilidad de los resultados, transformándose en una limitación para el desarrollo científico en esta área.

En este contexto, el cuestionario de Supervisión Abusiva de Tepper (SAT) (Tepper, 2000) destaca por ser uno de los instrumentos que ha recibido mayor atención por la comunidad científica, lo que se ha manifestado mediante su uso en distintos contextos culturales (Estados Unidos, Australia, China, Inglaterra, Philipinas, entre otros) (Martinko, Harvey, Brees and Mackey, 2013). Aunque esta escala es usada con frecuencia, existen muy pocos estudios que se enfoquen principalmente en el contraste de sus propiedades psicométricas, la mayoría presenta los indicadores de fiabilidad y validez reportados originalmente por Tepper (2000) y algunos obtienen evidencias preliminares ad hoc. Actualmente esta escala ha sido adaptada y validada para su uso en trabajadores chinos (Hu, Wu and Wang, 2011), italianos (Gatti, Caputo, and Cortese, 2020) y ecuatorianos (Moreno and Herrera, 2019). Estos estudios encontraron que los ítems poseen cargas factoriales satisfactorias $(\lambda>.6)$ y representan una estructura factorial unidimensional.

Esta carencia en la adaptación y validación del cuestionario de Supervisión Abusiva de Tepper
(SAT) es preocupante, sobre todo si se considera que gran parte de las investigaciones y organizaciones en Chile y Latinoamérica siguen utilizando escalas creadas $a d h o c$, sin evidencias de validez o adaptación a su contexto cultural, lo que tiene como consecuencia que la evidencia en la que se basa la toma de decisiones sea precaria o inexistente. Por tanto, ante la necesidad de contar con instrumentos que muestren apropiadas propiedades psicométricas específicas para cada cultura (AERA, APA and NCME, 2014; Muñiz, Elosua and Hambleton, 2013), tanto para la investigación como para el diagnóstico, prevención y tratamiento, este estudio tiene por objetivo adaptar lingüísticamente y validar el cuestionario de Supervisión Abusiva de Tepper (SAT) para su uso en adultos trabajadores de la ciudad de Arica, Chile, por medio de la obtención de evidencia de validez basada en la estructura interna del test y los coeficientes de fiabilidad alfa de Cronbach y omega jerárquico de McDonald's.

\section{MÉTODO}

\subsection{Diseño y participantes}

Este estudio tiene un diseño no experimental de tipo transversal e instrumental (Ato, López-García y Benavente, 2013).

Se utilizó una estrategia de muestreo no probabilística por conveniencia (Otzen y Manterola, 2017). La muestra está compuesta por 287 hombres provenientes de cuatro empresas privadas que pertenecen al sector pesquero y de la construcción en la región de Arica y Parinacota, zona urbana. De acuerdo con el tipo de trabajo, $245(85,4 \%)$ trabajadores declararon cumplir funciones operativas y 42 $(14,6 \%)$ administrativas. Los criterios de inclusión fueron los siguientes: (1) ser mayor de 18 años de edad; y (2) tener un supervisor directo a quien evaluar. No se aplicaron criterios de exclusión posteriores. Por solicitud de las empresas participantes, no se recolectó información sociodemográfica que permitiese identificar algún caso (e.g. edad, nivel socioeconómico, nacionalidad, tipo de contrato o sector de trabajo), garantizando de esta forma el anonimato de los trabajadores. Además, solo participaron trabajadores hombres, debido a que, durante la recolección de los datos, no se encontró presente ninguna trabajadora mujer. Esto se debe probablemente a que estos sectores han presentado una brecha de género histórica, en la que existe un número menor de mujeres trabajadoras como mano de obra permanente en comparación a la cantidad 
de hombres trabajadores (Comisión Económica para América Latina y el Caribe [CEPAL], 2019).

\subsection{Instrumentos}

El Cuestionario de Supervisión Abusiva de Tepper (SAT) (Tepper, 2000) consta de 15 ítems diseñados para medir la supervisión abusiva mediante una estructura factorial unidimensional. Esta escala contiene ítems que evalúan subjetivamente el grado en que los trabajadores perciben la supervisión de sus jefes (e.g. "Me recuerda los errores y fallas del pasado" o "No permite relacionarme con mis compañeros de trabajo"). Los ítems corresponden a declaraciones de comportamiento en una escala Likert de 5 niveles ( 1 = "No recuerdo" a 5 = "Siempre"). La escala original reportó adecuados niveles de fiabilidad $(\alpha>.90)$ y evidencias de validez basada en la estructura interna del test (Tepper, 2000). El protocolo de aplicación de la versión en español se muestra en el Anexo 1.

\subsection{Adaptación lingüística de la escala de supervisión abusiva}

Para adaptar la escala original de Supervisión Abusiva de Tepper (2000) se realizó un proceso de traducción hacia delante y hacia atrás (forward/ backward) de la versión en inglés. Inicialmente, la escala fue traducida por un experto de habla hispana, cuya adecuación lingüística y de contenido fue revisada mediante un acuerdo interjueces (i.e. dos doctores, un experto en psicometría y otro en psicología organizacional). Por último, un experto de habla inglesa tradujo la versión intermedia del español de nuevo al inglés (traducción hacia atrás) y comparó sus resultados con la versión original en inglés para asegurar la equivalencia conceptual, semántica y técnica (Kuyken et al., 1994). Debido a que los ítems de la escala de supervisión abusiva estaban escritos en un lenguaje claro, simple y directo, no fue necesario ajustar ningún ítem por diferencias culturales.

\subsection{Procedimiento}

Primeramente se contactó con gerentes de empresas privadas de Arica y se les invitó a participar voluntariamente en este estudio. Una vez obtenida la autorización, bajo el compromiso de devolución de un informe global y sin individualización de casos, se explicó a los trabajadores el propósito de la investigación, el carácter voluntario y anónimo de las respuestas, además de la disponibilidad para resolver cualquier pregunta o abandonar la aplicación en cualquier momento. Quienes accedieron a participar firmaron un consentimiento informado que expresó por escrito lo mencionado anteriormente. Luego se acordó una fecha de administración del cuestionario. La encuesta se aplicó en formato de lápiz y papel, durante una jornada de trabajo regular y el procedimiento de respuesta duró aproximadamente 5 minutos. Por último, el anonimato se resguardó mediante la devolución anónima en un sobre cerrado, sin ningún tipo de datos de identificación personal.

Esta investigación fue aprobada desde el punto de vista técnico y ético por el Comité de Carrera de Psicología de la Universidad de Tarapacá.

\subsection{Análisis de datos}

Análisis preliminares mostraron una escasa variabilidad en aquellas puntuaciones que indicaban algún tipo de presencia de abuso y una distribución de datos no normal (i.e. en la prueba de KolmogorovSmirnov, $p<.05$ ). Por tal razón se "dicotomizó" la escala entre ausencia y presencia (ver Anexo 2), donde el valor "no recuerdo" equivale "ausencia" (codificado como 0) y cualquier otro valor equivale a "presencia" (codificado como 1). Todo esto, con el fin de aumentar la capacidad de discriminación del instrumento y evitar la introducción de errores de medida (Abad, Olea, Ponsoda y García, 2011). Los análisis de datos se hacen a partir de los valores dicotomizados.

Para establecer evidencia de validez basada en la estructura interna del test, se utilizó un análisis factorial confirmatorio (AFC) con un método de estimación de mínimos cuadrados ponderados robustos (WLSMV), el que es robusto con variables discretas no normales. El análisis AFC se realizó a partir de matrices de correlaciones tetracóricas (e.g. Morata-Ramírez y Holgado-Tello, 2013), y el ajuste general del modelo se evaluó siguiendo las recomendaciones de punto de corte propuesto por Schreiber (2017) (CFI and TLI $>.90$ es aceptable y $>.95$ es satisfactorio; RMSEA $<.08$ es aceptable $\mathrm{y}<.06$ es satisfactorio).

Para establecer evidencia de fiabilidad, se utilizó los coeficientes alfa de Cronbach y omega jerárquico de McDonald's, donde valores sobre .70 son considerados aceptables y sobre .80 satisfactorios (Cho and Kim, 2015). Los análisis se realizaron con 
los programas estadísticos Mplus en su versión 8.2 (Muthén and Muthén, 1998-2017) y Jamovi en su versión 1.2 (The jamovi Project, 2020).

\section{RESULTADOS}

El modelo original (Tabla 1) presentó inadecuaciones para explicar la matriz de covariaciones observadas, con niveles de ajuste inferiores a los estándares más recomendados en la literatura (CFI > .95, TLI > .95, y RMSEA < .06) (Schreiber, 2017), por lo que se procedió a depurar iterativamente el modelo de medida, mediante la eliminación de 2 ítems que presentaron redundancias (Abad, Olea,
Ponsoda y García, 2011) o cargas factoriales débiles $(\lambda<.3)$ (Cohen, 1988), obteniendo de esta forma una escala más breve y optimizada. Finalmente, los indicadores de ajuste comparativos (e.g. CFI; TLI) y absolutos (e.g. x2/gl; RMSEA) de la escala depurada de 13 ítems sugieren que el modelo unidimensional es una adecuada representación poblacional de la matriz de covariaciones observadas. Los detalles del ajuste global de los modelos de medida se presentan en la Tabla 1.

En la Tabla 2 se observa que la versión depurada de la escala de Supervisión Abusiva de Tepper (SAT) tiene cargas factoriales fuertes

\section{Tabla 1}

Indicadores de ajuste global de los modelos de medida

\begin{tabular}{lccccccccc}
\hline Modelos & Par & \multirow{2}{*}{$\chi^{2}$} & $\mathrm{gl}$ & $\mathrm{P}$ & $\mathrm{CFI}$ & $\mathrm{TLI}$ & RMSEA & \multicolumn{2}{c}{ RMSEA IC 90\% } \\
\hline SAT Dicotomizado & 30 & 180.783 & 90 & .000 & .973 & .969 & .059 & .047 & .072 \\
SAT Dicotomizado* $^{*}$ & 26 & 95.057 & 65 & .008 & .990 & .988 & .040 & .021 & .057 \\
\hline
\end{tabular}

Nota: Par = Número de parámetros; $\chi 2=$ Chi cuadrado $g l=$ Grados de libertad $; p=$ Significado CFI $=$ Índice de ajuste comparativo; TLI = Índice de Tucker-Lewis; RMSEA = Error del cuadrado medio de la raíz de aproximación. IC = Intervalo de confianza; Mín. = Mínimo; Máx. = Máximo; $*$ = Modelo depurado.

Tabla 2

Cargas factoriales estandarizadas y coeficientes de fiabilidad

\begin{tabular}{lc}
\hline Ítems & SAT \\
\hline Me dice que lo que pienso o siento es estúpido & $.819^{* *}$ \\
Se queda callado cuando le hago una pregunta & $.648^{* *}$ \\
Se burla de mí, delante de los demás & $.826^{* *}$ \\
Invade mi privacidad & $.804^{* *}$ \\
Me recuerda los errores y fallas del pasado & $.730^{* *}$ \\
No le importa los trabajos que me han llevado un gran esfuerzo & $.753^{* *}$ \\
Me culpa a mí para salvarse a sí mismo de la vergüenza & $.823^{* *}$ \\
Expresa su rabia hacia mí cuando se enoja con otra persona & $.763^{* *}$ \\
Hace comentarios negativos sobre mí a los demás & $.862^{* *}$ \\
Es grosero conmigo & $.916^{* *}$ \\
No permite relacionarme con mis compañeros de trabajo & $.779^{* *}$ \\
Me llama incompetente & $.968^{* *}$ \\
Me miente & $.760^{* *}$ \\
Estimadores de fiabilidad & .879 \\
Alpha $(\alpha)$ & .887 \\
Omega $(\omega)$ & \\
\hline
\end{tabular}

Nota: Alpha $(\alpha)=$ Coeficiente alfa de Cronbach; Omega $(\omega)=$ Coeficiente omega jerárquico de McDonald's; Las cargas factoriales $>.5$ están en negrita; $* * \mathrm{p}<.01 ; * \mathrm{p}<.05$. 
$(\lambda>.50)$, lo que sugiere que los ítems son buenas representaciones de la variable latente. Adicionalmente se observa que las estimaciones de fiabilidad fueron satisfactorias $(\alpha>.80)$ o al menos suficientes ( $\alpha$ $>$.70), según se utilice alfa de Cronbach u omega jerárquico de McDonald's.

\section{DISCUSIÓN}

El propósito de esta investigación fue adaptar lingüísticamente y validar el cuestionario de Supervisión Abusiva de Tepper (SAT) para su uso en adultos trabajadores de la ciudad de Arica, Chile, mediante la obtención de evidencia de validez basada en la estructura interna del test y los coeficientes de fiabilidad alfa de Cronbach y omega jerárquico de McDonald's. De acuerdo con los indicadores de ajuste del modelo depurado y al tamaño de las cargas factoriales, este estudio proporciona suficiente evidencia de validez basada en la estructura interna del test para sostener la estructura unidimensional de la versión dicotomizada. Estos hallazgos son consistentes con los estudios previos que contrastaron sus propiedades psicométricas (Hu, Wu and Wang, 2011; Gatti, Caputo, y Cortese, 2020; Moreno y Herrera, 2019). Del mismo modo, las estimaciones de los coeficientes de fiabilidad permiten suponer que la estructura factorial posee una coherencia interna adecuada, que minimiza los errores de medición.

La escasa variabilidad que presenta la versión Likert de la escala, nos hace sugerir utilizar la versión "dicotomizada", ya que, al parecer, sería más fácil de reconocer que una conducta manifiesta al diferenciar su frecuencia de ocurrencia. Se ha señalado que la supervisión abusiva no es algo frecuente (Tepper, Simon and Park, 2017) y que las frecuencias reportadas pueden estar subestimadas por temor a perder el trabajo, ser enjuiciados o ser reconocidos como víctimas (Tepper, Duffy, Henle and Lambert, 2006) o sobrestimadas por la tendencia de algunos sujetos de reconocer como abuso algunos comportamientos no abusivos (Brees, Martinko and Harvey, 2016). Otra razón responde probablemente a la cultura organizacional de Chile, en la que los subordinados al asumir acríticamente las opiniones e instrucciones tiendan a subestimar o no reconocer las conductas indeseadas y hostiles de sus supervisores. Estos comportamientos laborales conformistas, disciplinados y obedientes son determinados por prácticas administrativas caracterizadas por sus dificultades para delegar la autoridad, descentralizar y distribuir el poder en la toma de decisiones (Didier y Luna, 2017).

La principal restricción de este estudio corresponde a la utilización de una estrategia de muestreo no probabilística (i.e. por conveniencia) en donde la elección de los sujetos no fue al azar, por tanto, la generabilidad de los valores poblaciones estimados en este estudio deben ser considerados con precaución. Se sugiere realizar nuevos estudios que evalúen las propiedades psicométricas en otros contextos y áreas laborales con el fin de aumentar la posibilidad de generalización. Asimismo, como las propiedades psicométricas del cuestionario de Supervisión Abusiva de Tepper (SAT) se obtuvieron a partir de una transformación escalar de formato Likert (i.e. 1-5) a dicotómica (i.e. 0-1) y no de una aplicación directa, es preciso nuevos estudios empíricos que estimen su evidencia de validez y fiabilidad, a partir de la aplicación directa de la forma dicotómica. En este sentido, para complementar los hallazgos de este estudio, también es necesario desarrollar investigaciones en la que se obtenga evidencia de validez basada en la relación con otras variables y se realicen invarianzas por sexo, funciones (operativa y administrativa) y tipo de empresa u organización.

Finalmente, a pesar de las limitaciones, esta escala debe ser incorporada en protocolos de evaluación o en baterías de medición para su uso en los servicios de salud laboral y de protección de riesgos psicosociales (i.e. SUSESO/ISTAS 21) de Chile, ya que permitirá evaluar la necesidad de intervenciones preventivas que mejoren el bienestar y rendimiento de los trabajadores y reduzcan las consecuencias psicológicas asociadas al acoso y abandono laboral. En consecuencia, se podrá mejorar y complementar el diseño de estrategias que promuevan relaciones supervisor-colaborador saludables. Los hallazgos de este estudio sugieren que el cuestionario de Supervisión Abusiva de Tepper (SAT) en su versión "dicotomizada", puede ser utilizado para el desarrollo de investigaciones junto con otras variables psicológicas que estén implicadas en la salud mental de los trabajadores, liderazgo, clima y productividad laboral.

\section{CONCLUSIÓN}

Los resultados de este estudio proporcionan evidencia suficiente para la adaptación lingüística y validación del cuestionario de Supervisión Abusiva de Tepper (SAT) en trabajadores de Chile, mediante 
indicadores de fiabilidad y validez basada en la estructura interna del test, que dan soporte a la interpretación de sus puntuaciones en muestras equivalentes. Estos hallazgos sugieren que la escala puede ser utilizada por profesionales de los servicios de salud laboral, protección de riesgos psicosociales y para el desarrollo de investigación en el área organizacional. Se recomienda utilizar el instrumento en su versión "dicotomizada". 


\section{REFERENCIAS}

Abad, F., Olea, J., Ponsoda, V., y García, C. (2011). Medición en ciencias sociales y de la salud. Madrid, España: Síntesis.

Ahmad, H., and Ibrahim, B. (2015). Leadership and the characteristic of different generational cohort towards job satisfaction. Procedia-Social and Behavioral Sciences, 204, 14-18. https:// doi.org/10.1016/j.sbspro.2015.08.104.

American Educational Research Association, American Psychological Association, and National Council on Measurement in Education. (2014). Standards for educational and psychological testing. Washington, DC: American Educational Research Association

Ashforth, B. (1987). Organizations and the petty tyrant: An exploratory study. Paper presented at the annual meeting of the Academy of Management, New Orleans, LA.

Ashforth, B. (1994). Petty tyranny in organizations. Human relations, 47(7), 755-778. https://doi.org/10.1177/00187267 9404700701.

Ato, M., López-García, J., y Benavente, A. (2013). Un sistema de clasificación de los diseños de investigación en psicología. Anales de Psicología, 29(3), 1038-1059. https://doi.org/10.6018/ analesps.29.3.178511.

Boddy, C. (2011). Corporate psychopaths, bullying and unfair supervision in the workplace. Journal of Business Ethics, 100(3), 367-379. doi:10.1007/s10551-010-0689-5

Brees, J., Martinko, M., and Harvey, P. (2016). Abusive supervision: subordinate personality or supervisor behavior? Journal of Managerial Psychology, 31(2), 405-419. https://doi.org/10.1108/ JMP-04-2014-0129.

Burke, R. (2017). Toxic leaders: Exploring the dark side. Effective Executive, 20(1), 10.

Burns, W. (2017). A descriptive literature review of harmful leadership styles: Definitions, commonalities, measurements, negative impacts, and ways to improve these harmful leadership styles. Creighton Journal of Interdisciplinary Leadership, 3(1), 33-52. http://dx.doi.org/10.17062/cjil.v3i1.53.

Chen, Z., and Wang, H. (2017). Abusive supervision and employees' job performance: A multiple mediation model. Social Behavior and Personality: an international journal, 45(5), 845-858. https://doi.org/10.2224/sbp.5657.

Cho, E., and Kim, S. (2015). Cronbach's coefficient alpha: Wellknown but poorly understood. Organizational Research Methods, 18(2), 207-230. https://doi.org/10.1177/1094428114555994.

Comisión Económica para América Latina y el Caribe [CEPAL] (2019). Indicadores que visibilizan las brechas de género en el mercado laboral. Editorial Naciones Unidas.

Contreras, F., Barbosa, D., Juárez, F., Uribe A. y Mejía, C. (2009). Estilos de Liderazgo, Clima Organizacional y Riesgos Psicosociales en Entidades del Sector Salud. Un Estudio Comparativo. Acta Colombiana de Psicología, 12(2), 13 - 26.

De Jong, J., and Den Hartog, D. (2007). How leaders influence employees' innovative behaviour. European Journal of innovation management, 10(1), 41-64. https://doi. org/10.1108/14601060710720546.

Didier, N., y Luna, J. (2017). ¿Dónde estamos? La cultura laboral chilena desde Hofstede. Revista Colombiana de Psicología, 26(2), 295-311.
Farh, C. and Chen, Z. (2014). Beyond the Individual Victim: Multilevel Consequences of Abusive Supervision in Teams. Journal of Applied Psychology, 99(6), 1074-1095. http://dx.doi. org/10.1037/a0037636.

Gatti, P., Caputo, A., and Cortese, C. (2020). An initial validation of Tepper's Abusive Supervision Scale. BPA-Applied Psychology Bulletin (Bollettino di Psicologia Applicata), 67(284).

Gómez, C. and Rodríguez, J. (2006). Four keys to Chilean culture: Authoritarianism, legalism, latalism and compadrazgo. Asian Journal of Latin American Studies, 3, 43-65.

Hofstede, G. and Minkov, M. (2010). Long- versus short-term orientation: New perspectives. Asian Pacific Business Review, 16(4), 493-504. https://doi.org/10.1080/13602381003637609

$\mathrm{Hu}, \mathrm{C} ., \mathrm{Wu}, \mathrm{T}$., and Wang, Y. (2011). Measurement equivalence/ invariance of the abusive supervision measure across workers from Taiwan and the United States. The Journal of Psychology: Interdisciplinary and Applied,145, 111-131.

Instituto Nacional de Estadística [INE] (2021). Boletín estadístico: Empleo Trimestral, edición $n .^{\circ}$ 269, 31 de marzo 2021. Instituto Nacional de Estadística, Chile.

Khan, S., Qureshi, I. and Ahmad, H. (2010). Abusive Supervision and Negative Employee Outcomes. European journal of social sciences, $15(4), 490-500$.

Krasikova, D., Green, S., and LeBreton, J. (2013). Destructive leadership a theoretical review, integration, and future research agenda. Journal of Management, 39(5), 1308-338. https://doi. org/10.1177/0149206312471388.

Kuyken, W., Orley, J., Hudelson, P., and Sartorius, N. (1994). Quality of Life Assessment across Cultures. International Journal of Mental Health, 23(2), 5-27. https://doi.org/10.1080 /00207411.1994.11449281.

Liang, L., Lian, H., Brown, D., Ferris, D., Hanig, S., and Keeping, L. (2016). Why are abusive supervisors abusive? A dual-system self-control model. Academy of Management Journal, 59(4), 1385-1406. https://doi.org/10.5465/amj.2014.0651.

Mackey, J., Frieder, R., Brees, J., and Martinko, M. (2017). Abusive supervision: A meta-analysis and empirical review. Journal of Management, 43(6), 1940-1965. https://doi. org/10.1177/0149206315573997.

Martinko, M., Harvey, P., Brees, J., and Mackey, J. (2013). A review of abusive supervision research. Journal of Organizational Behavior, 34(1), 120-137. https://doi.org/10.1002/job.1888.

Martinko, M., Harvey, P., Sikora, D., and Douglas, S. (2011). Perceptions of abusive supervision: The role of subordinates' attribution styles. The Leadership Quarterly, 22(4), 751 - 764. https://doi.org/10.1016/j.leaqua.2011.05.013.

Morata-Ramírez, M., and Holgado-Tello, F. (2013). Construct validity of Likert scales through Confirmatory Factor Analysis: A Simulation study comparing different methods of estimation based on Pearson and polychoric correlations. International Journal of Social Science Studies, 1(1), 54-61. https://doi. org/10.11114/ijsss.v1i1.27.

Moreno, J., Herrera, D., y Gargurevich, R. (2019). Propiedades Psicométricas de la Versión en Español de la Escala de Supervisión Abusiva en Población Laboral Ecuatoriana. Revista iberoamericana 
de diagnóstico y evaluación psicológica, 2(51), 117-134. https:// doi.org/10.21865/RIDEP51.2.09.

Muñiz, J., Elosua, P., and Hambleton, R. (2013). International Test Commission Guidelines for test translation and adaptation: Second edition. Psicothema, 25(2), 151-157. https://doi. org/10.7334/psicothema2013.24

Muthén, L. and Muthén, B. (1998-2017). Mplus User's Guide, Eighth Edition. Los Ángeles, CA: Muthén \& Muthén.

Otzen, T., y Manterola, C. (2017). Técnicas de Muestreo sobre una Población a Estudio. International Journal of Morphology, 35(1), 227-232. http://dx.doi.org/10.4067/S0717-95022017000100037.

Palomino, J., Espinoza, J. y Arellano, M. (2013). Clima Laboral: La Influencia de la Supervisión, Los Factores Organizacionales y La Predisposición de Los Empleados. Revista Internacional Administración \& Finanzas, 6(6), 55-71.

Raskin, R., and Hall, C. (1979). A narcissistic personality inventory. Psychological Reports, 45, 590. https://doi.org/10.2466/ pr0.1979.45.2.590

Rodwell, J., Brunetto, Y., Demir, D., Shacklock, K., and FarrWharton, R. (2014). Abusive supervision and links to nurse intentions to quit. Journal of Nursing Scholarship, 46(5), 357365. https://doi.org/10.1111/jnu.12089.

Rosenthal, S., and Pittinsky, T. (2006). Narcissistic leadership. The leadership quarterly, 17(6), 617-633. https://doi.org/10.1016/j. leaqua.2006.10.005.

Schmidt, A. (2008). Development and validation of the Toxic Leadership Scale. (Tesis de Maestría en Ciencias), University of Maryland, College Park. http://drum.lib.umd.edu/bitstream/1903/8176/1/umi-umd-5358.pdf.

Schreiber, J. (2017). Update to core reporting practices in structural equation modeling. Research in Social and Administrative Pharmacy, 13(3), 634-643. https://doi.org/10.1016/j. sapharm.2016.06.006.
Tepper, B. (2000). Consequences of Abusive Supervision. Academy of Management Journal, 43(2), 178-190. https://doi. org/10.5465/1556375.

Tepper, B. (2007). Abusive supervision in work organizations: Review, synthesis, and research agenda. Journal of management, 33(3), 261-289. https://doi.org/10.1177/0149206307300812.

Tepper, B., Duffy, M., Henle, C., and Lambert, L. (2006). Procedural injustice, victim precipitation, and abusive supervision. Personnel Psychology, 59(1), 101-123. https://doi. org/10.1111/j.1744-6570.2006.00725.x.

Tepper, B., Simon, L., and Park, H. (2017). Abusive supervision. Annual Review of Organizational Psychology and Organizational Behavior, 4, 123-152. https://doi.org/10.1146/ annurev-orgpsych-041015-062539.

The jamovi project (2020). jamovi (Version 1.2) [Computer Software]. Retrieved from https://www.jamovi.org

Vogel, R., Mitchell, M., Tepper, B., Restubog, S., Hu, C., Hua, W., and Huang, J. (2015). A cross-cultural examination of subordinates' perceptions of and reactions to abusive supervision. Journal of Organizational Behavior, 36(5), 720-745. https://doi. org/10.1002/job.1984.

Zhang, Y., and Bednall, T. (2016). Antecedents of abusive supervision: A meta-analytic review. Journal of Business Ethics, 139(3), 455-471. https://doi.org/10.1007/s10551-015-2657-6.

Zhang, Y., and Liao, Z. (2015). Consequences of abusive supervision: A meta-analytic review. Asia Pacific Journal of Management, 32(4), 959-987. https://doi.org/10.1007/s10490-015-9425-0.

Zhou, B., Marchand, A., and Guay, S. (2018). Gender differences on mental health, work-family conflicts and alcohol use in response to abusive supervision. Revista de Psicología del Trabajo y de las Organizaciones, 34(3), 157-167. https://doi. org/10.5093/jwop2018a18. 


\section{Anexo 1. Protocolo de aplicación de la escala de Supervisión Abusiva de Tepper (SAT)}

Estimado(a) Trabajador(a):

A continuación se presentan varias declaraciones relacionadas con las conductas de su supervisor. Por favor, piense en su Jefe/Supervisor Directo (si tiene más de uno, piense en el que tiene más contacto directo con usted) y las conductas que tiene hacia usted y señale, en cada enunciado, qué tan frecuente/ común su Jefe tiene o ha tenido esa conducta hacia usted. Indique con una "X" la frecuencia que mejor represente su realidad:

Por ejemplo, si la pregunta fuera "me llama la atención inadecuadamente" y su jefe nunca ha tenido esa conducta, marque con una "X" la alternativa "No recuerdo", pero si lo hace todos los días o con muchísima frecuencia, señale "Siempre".

\begin{tabular}{|c|c|c|c|c|c|c|}
\hline N. ${ }^{\circ}$ & Mi Jefe o Supervisor Directo: & $\begin{array}{l}\text { No } \\
\text { recuerdo }\end{array}$ & Raramente & Ocasionalmente & $\begin{array}{l}\text { Casi } \\
\text { siempre }\end{array}$ & Siempre \\
\hline 1 & Se burla de mí & & & & & \\
\hline 2 & Me dice que lo que pienso o siento es estúpido & & & & & \\
\hline 3 & Se queda callado cuando le hago una pregunta & & & & & \\
\hline 4 & Se burla de mí, delante de los demás & & & & & \\
\hline 5 & Invade mi privacidad & & & & & \\
\hline 6 & Me recuerda los errores y fallas del pasado & & & & & \\
\hline 7 & $\begin{array}{l}\text { No le importa los trabajos que me han llevado } \\
\text { un gran esfuerzo }\end{array}$ & & & & & \\
\hline 8 & $\begin{array}{l}\text { Me culpa a mí para salvarse a sí mismo de } \\
\text { la vergüenza }\end{array}$ & & & & & \\
\hline 9 & Rompe las promesas hechas & & & & & \\
\hline 10 & $\begin{array}{l}\text { Expresa su rabia hacia mí cuando se enoja } \\
\text { con otra persona }\end{array}$ & & & & & \\
\hline 11 & $\begin{array}{l}\text { Hace comentarios negativos sobre mí a los } \\
\text { demás }\end{array}$ & & & & & \\
\hline 12 & Es grosero conmigo & & & & & \\
\hline 13 & $\begin{array}{l}\text { No permite relacionarme con mis compa- } \\
\text { neros de trabajo }\end{array}$ & & & & & \\
\hline 14 & Me llama incompetente & & & & & \\
\hline 15 & Me miente & & & & & \\
\hline
\end{tabular}


Anexo 2. Análisis preliminares acerca de la variabilidad de las puntuaciones de la escala SAT

Tabla 3

Estadísticos descriptivos de la escala SAT en formato Likert y Dicotómico

\begin{tabular}{ccc}
\hline \multirow{2}{*}{. $^{\circ}$ ítems } & Likert & Dicotomizado \\
\cline { 2 - 3 } & $\mathrm{M}(\mathrm{DE})$ & $\%(\mathrm{~N})$ \\
\hline 1 & $1,35(0,74)$ & $22,3 \%(64)$ \\
2 & $1,24(0,62)$ & $16,7 \%(48)$ \\
3 & $1,60(0,99)$ & $39,9 \%(114)$ \\
4 & $1,32(0,74)$ & $19,2 \%(55)$ \\
5 & $1,38(0,86)$ & $21,3 \%(61)$ \\
6 & $1,73(1,05)$ & $42,9 \%(123)$ \\
7 & $1,90(1,32)$ & $43,0 \%(123)$ \\
8 & $1,47(0,96)$ & $25,1 \%(72)$ \\
9 & $1,65(1,25)$ & $32,7 \%(93)$ \\
10 & $1,53(1,06)$ & $26,1 \%(75)$ \\
11 & $1,39(0,97)$ & $19,2 \%(55)$ \\
12 & $1,28(0,84)$ & $14,3 \%(41)$ \\
13 & $1,41(1,00)$ & $20,6 \%(59)$ \\
14 & $1,18(0,75)$ & $7,3 \%(21)$ \\
15 & $1,37(0,85)$ & $20,6 \%(59)$ \\
Total & $1,43(0,61)$ & - \\
\hline
\end{tabular}

Nota: . $^{\circ}$ ítems = Número de ítems M = Media; DE = Desviación estándar; \% = Porcentaje; $\mathrm{N}=$ Cantidad de sujetos. 